Note: This is a draft of a paper submitted for publication. Contents of this paper should not be quoted or referred to without permission of the author(s).

Presented at Fifth International Conference on Polycrystalline Semiconductor

Schwäbisch-Gmünd, Germany, September 13-18, 1998 and published in

Polycrystalline Semiconductors V-Bulk Materials, Thin Films, and Devices, ed. by J. H. Werner, H. P. Strunk, and H. W. Schock, Trans Tech Publications Ltd., Winterhur, Switzerland

\title{
Atomic Structure and Properties of Extended Defects in Silicon
}

M. F. Chisholm, ${ }^{1}$ R. Buczko, ${ }^{1,2}$ M. Mostoller, ${ }^{1}$ T. Kaplan, ${ }^{1}$ A. Maiti,, 2

S. T. Pantelides, ${ }^{1,2}$ and S. J. Pennycook ${ }^{1}$

1 Solid State Division, Oak Ridge National Laboratory

P.O. Box 2008, Oak Ridge, Tennessee 37831-6030

2Department of Physics and Astronomy, Vanderbilt, University

Nashville, Tennessee 37235

\author{
prepared by \\ SOLID STATE DIVISION \\ OAK RIDGE NATIONAL LABORATORY \\ Managed by \\ LOCKHEED MARTIN ENERGY RESEARCH CORP. \\ under \\ Contract No. DE-AC05-96OR22464 \\ with the
}

U.S. DEPARTMENT OF ENERGY

Oak Ridge, Tennessee

October 1998 


\section{DISCLAIMER}

This report was prepared as an account of work sponsored by an agency of the United States Government. Neither the United States Government nor any agency thereof, nor any of their employees, make any warranty, express or implied, or assumes any legal liability or responsibility for the accuracy, completeness, or usefulness of any information, apparatus, product, or process disclosed, or represents that its use would not infringe privately owned rights. Reference herein to any specific commercial product, process, or service by trade name, trademark, manufacturer, or otherwise does not necessarily constitute or imply its endorsement, recommendation, or favoring by the United States Government or any agency thereof. The views and opinions of authors expressed herein do not necessarily state or reflect those of the United States Government or any agency thereof. 


\section{DISCLAIMER}

Portions of this document may be illegible in electronic image products. Images are produced from the best available original document. 


\title{
ATOMIC STRUCTURE AND PROPERTIES OF EXTENDED DEFECTS IN SILICON
}

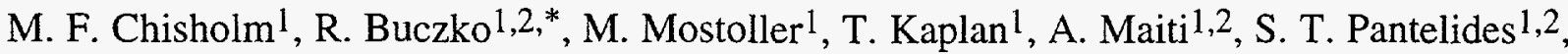 \\ and S. J. Pennycook ${ }^{1}$ \\ ${ }^{1}$ Solid State Division, Oak Ridge National Laboratory, Oak Ridge, TN 37831-6030, USA \\ 2Department of Physics and Astronomy, Vanderbilt University, Nashville, TN 37235, USA
}

Keywords: Z-contrast, transmission electron microscopy, electronic structure, dislocations, grain boundaries

\begin{abstract}
The Z-contrast technique represents a new approach to high-resolution electron microscopy allowing for the first time incoherent imaging of materials on the atomic scale. The key advantages of the technique, an intrinsically higher resolution limit and directly interpretable, compositionally sensitive imaging, allow a new level of insight into the atomic configurations of extended defects in silicon. This experimental technique has been combined with theoretical calculations (a combination of first principles, tight binding, and classical methods) to extend this level of insight by obtaining the energetics and electronic structure of the defects.
\end{abstract}

\section{Introduction}

In the past half century, the world has been transformed by an electronic revolution that is largely based on silicon technology. Semiconductor devices have permeated all sectors of society and the economy in the industrialized world. Since the transistor was invented in 1948, semiconductor devices have undergone continuous miniaturization that has resulted in vastly improved utility accompanied by tremendous reductions in cost. Another notable technological success has been in the growth of defect-free semiconductor materials. The industry will soon be using highly perfect (structure and composition) silicon wafers with diameters up to $300 \mathrm{~mm}$.[1] Microelectronics, device miniaturization, and perfect single crystal substrates have been inseparably linked. However, the perfect semiconductor single crystal is not very useful; it is the defects that dramatically modify the material's electrical conductivity and carrier lifetimes over wide ranges. These defects have both vital and fatal consequences on semiconductors [2]; it is the ability to understand and control the defects that determines their desirability and utility.

This paper will present some recent examples where we have combined atomic resolution microscopy and computational techniques to understand and correlate the atomic and electronic structures of extended defects (grain boundaries and dislocations) in silicon.

\section{Z-contrast imaging}

When exploring the unknown, we rely heavily on our eyes to provide a direct image of the new object. The majority of objects we encounter are illuminated by large incoherent sources, such as a fluorescent light tube. Light reflecting from one part of the object is unable to coherently interfere with light reflecting from a different part of the object, and the resulting incoherent image is straightforward to interpret. If the only light sources available to us were coherent (such as laser beams) the confusing mass of fringes and other coherent interference effects, while containing much information on the object, would be practically impossible to interpret. In order to explore the unforeseen atomic configurations present at extended defects in materials it, again, would be desirable if one could obtain a directly interpretable incoherent image of the unfamiliar structures present in the defect cores. Z-contrast transmission electron microscopy provides such a view with both atomic resolution and compositional sensitivity.[3] 
Lord Rayleigh was the first to consider incoherent imaging for light microscopy.[4] He noted that by illuminating an object with a large incoherent source, the confusing interference features were suppressed and that there was also a doubling of the resolving power of the microscope compared to the coherent mode. However, up to the present time, most high resolution transmission electron microscopy has concentrated on trying to achieve the highest degree of coherence possible in the illuminating beam, and then trying to match the resulting complicated image intensity to simulated images from trial structures. The danger with this image matching approach is that the correct structure may be overlooked. In a conventional transmission electron microscope (TEM), however, it is not straightforward to form a large incoherent electron beam.

The development of an atomic resolution scanning transmission electron microscope (STEM) has now opened to door to incoherent transmission electron microscopy. A STEM operates as a conventional TEM with the optics reversed. The lens is placed before the specimen and focuses an electron probe of atomic dimensions which is scanned over the specimen. The $100 \mathrm{kV}$ and $300 \mathrm{kV}$ microscopes located at the Oak Ridge National Laboratory are capable of producing electron probes of $0.22 \mathrm{~nm}$ and $0.13 \mathrm{~nm}$ diameter, respectively; these probe diameters determine the resolution of the technique. Instead of a large incoherent source, we now need to use a large detector to collect the electrons, and the total intensity of electrons detected is displayed as a function of probe position. As long as the geometry of the detector is larger than any coherent interference features formed by the specimen, the image can be treated as being incoherent and the imaged atomic columns can be treated as independent scatterers. There is a direct correspondence between the object and its image and there are no contrast reversals with sample thickness or objective lens defocus.[5]

Using a large high-angle annular dark-field detector we collect only the high-angle electron scattering produced close to the atom nucleus. The intensity contributed to the image by each atomic column is therefore strongly dependent on the atomic numbers of the atoms present, providing strong compositional sensitivity, hence the name Z-contrast. Additionally at higher collection angles, the degree of thermal diffuse scattering in the electron scattering increases, helping to decrease the coherence length along the atomic columns.[6] The scattered intensity approaches the compositional sensitivity of the atomic numbered squared dependence of Rutherford scattering and allows detection of compositional inhomogenieties, for example, impurities segregated to the defect cores.

One of the most fundamental questions that must be addressed in developing a microscopic understanding of defects in silicon is their atomic configuration. Using the Z-contrast technique we have the ability to directly image and interpret unforeseen structures of the defect cores.

\section{The theoretical method}

Using the data from the Z-contrast image as the initial input and as a constraint on the possible structures, theoretical calculations were used to obtain the relaxed structure of the extended defects, the effects of segregated impurities, and the energy levels in the gap region that influence the electronic and optical properties. Calculations of the atomic structures discussed herein were performed using density functional theory and the local-density approximation for exchange and correlation.[7] Density functional theory is currently the premier tool for first-principles predictions of stable atomic configurations, dynamic rearrangements induced by internal or external forces, and the corresponding electrical and optical properties. The atomic cores were represented by nonlocal, norm-conserving pseudopotentials of the Kerker type.[8] The integration over the Brillouin zone was performed using various numbers of special $k$ points chosen using the Monkhorst-Pack scheme.[9] The electronic wave functions were expanded in a plane wave basis set with an energy cutoff of 150$200 \mathrm{eV}$. The self-consistent electron density was determined by the conjugate gradient scheme of Payne et al.[10] and the atoms were moved until the largest force on any atom was less than 0.08 eV/A.

It is well known that LDA calculations give values for the valence-to-conduction band gaps that are too small. To investigate the effects of dislocations on the electronic structure, we used the tight-binding method using the $s p^{3} s$ model of Vogl et al.[11], which gives acceptable values for the band gaps in the group-IV and III-V semiconductors. Atomic coordinates were taken from supercells relaxed by LDA calculations. 


\section{Models of grain boundaries}

There are two general categories of grain boundary models. One view is what we shall call the unruly crowd models originated by Quincke, who suggested the presence of an amorphous cement between grains.[12] The other view is that grain boundaries are best described as a wellordered zone between the lattices of two adjacent grains. Low angle grain boundaries composed of an array of lattice dislocations became the defining structures of these models since the original suggestions by Taylor [13] and Burgers.[14] However, at misorientations greater than about $15^{\circ}$, the separation of the required dislocations becomes so small that the description was thought to become unphysical.

Instead, most high angle grain boundary models, at present, incorporate more complicated but still ordered transition zones to accommodate the misorientation. The most complete and accepted classification is the structural unit model of Sutton and Vitek.[15] However, as pointed out by Bishop and Chalmers in an earlier description of the structural unit model, the three descriptions of a grain boundary (an array of coincidence atoms, structural units, or dislocations) are in some sense equivalent.[16]

We will use the dislocation description, for as the following images demonstrate, the dislocation model remains a reasonable description of the atomic configurations observed in tilt grain boundaries in silicon, even at high tilt angles.

\section{[110] tilt boundaries}

One of the most widespread applications of high resolution phase contrast electron microscopy has been the characterization of diamond cubic semiconductors. Much of this attention has focused on the relatively open $\langle 110\rangle$ projection of this lattice and on its defects. In fact, in the pioneering high resolution microscopy study of germanium published in 1977, Krivanek et al. correctly determined the atomic structure of a high angle $<110>$ tilt boundary. [17]

Figure 1 is an image of the corresponding $39^{\circ}<110>$ symmetric tilt boundary $(\Sigma=9\{122\}$ $<110>$ ) in silicon as viewed using the Z-contrast technique. The atomic column positions are (always) the bright features in the Z-contrast image. In this $\langle 110\rangle$ projection, diamond cubic crystals contain closely spaced pairs of atomic columns commonly referred to as dumbbells. In silicon, the projected separation of these two columns is $0.136 \mathrm{~nm}$ and as seen in the image they are resolved. The grain boundary, the region in which the misorientation of the two grains is accommodated, is seen to be extremely narrow. In fact there are no dumbbells in intermediate positions.

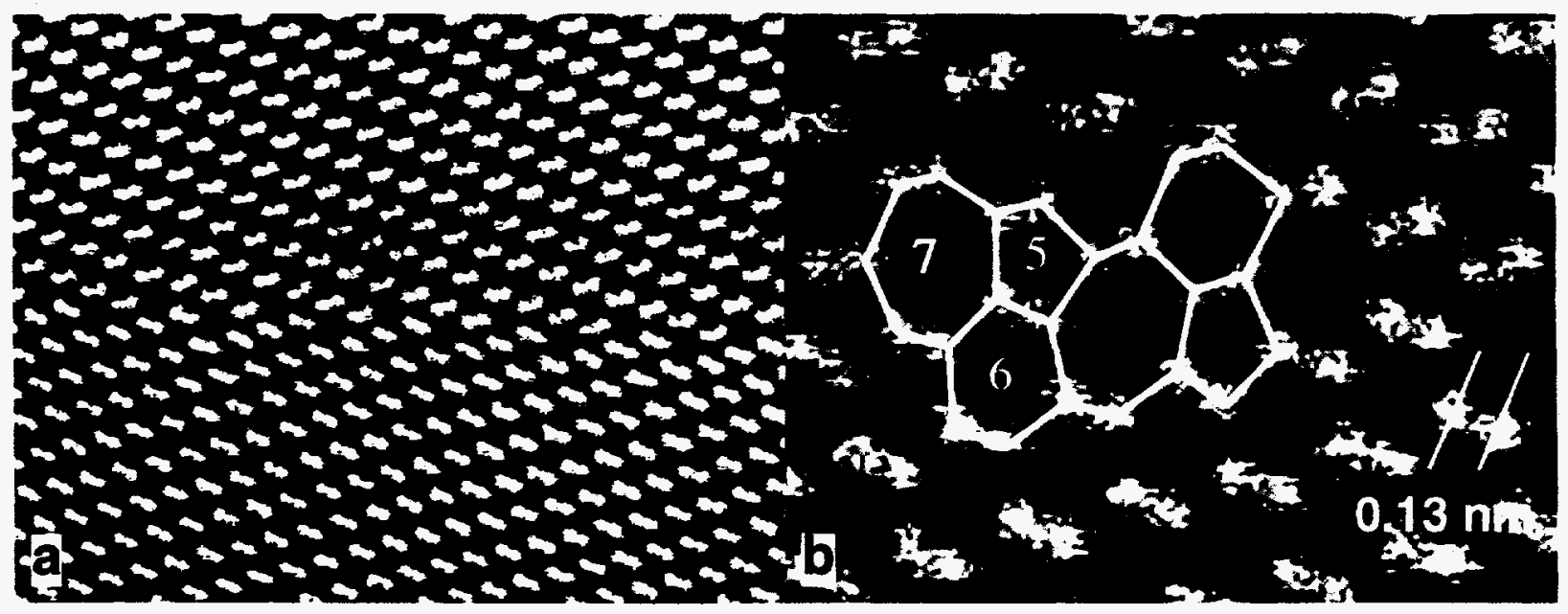

Figure 1. (a) Z-contrast image of a symmetric $39^{\circ} \Sigma=9\{221\}<110>$ silicon tilt grain boundary as viewed along the $\langle 110\rangle$ tilt axis. (b) A higher magnification view of the boundary showing the connected string of 5- and 7-membered ring structures present at its core. The separation between the parallel white lines in the images is $0.13 \mathrm{~nm}$. 
The boundary contains a continuous string of 5- and 7-membered ring structures in place of the 6-membered rings of the perfect diamond cubic lattice. This defect structure is identical to the core structure of a perfect edge dislocation with a $<110>$ line direction and a $a / 2<-110>$ Burgers vector. The structure of this Lomer dislocation was first predicted by Hornstra in the 1950's. [18] There are no dangling bonds seen in this boundary. Bulk-like tetrahedral bonding is maintained in the cores with relatively little distortion to the bond lengths and angles.

It is important to note that these dislocation cores can be packed one on top of the other and still maintain their distinct structure (see Fig. 1). These edge dislocations have been found to be one of only two defects present in $\mathrm{Si}<110>$ tilt boundaries. The other unit is the twin unit, a variation of the bulk 6-membered ring. It should then follow if we can unravel the secrets of this dislocation we will begin to unravel the secrets of boundaries with a $<110>$ tilt component as well.

\section{[001] tilt boundaries}

There have been far fewer high resolution investigations of $<001>$ tilt grain boundaries in diamond cubic semiconductors. The principle reason for this is that instruments with point-to-point resolution better than $0.15 \mathrm{~nm}$ are required to reveal the structural features in these boundaries. Hornstra, again, predicted the building blocks contained in these boundaries, long before microscopy had the tools to actually see them.[19] He proposed that any $<001>$ tilt boundary in diamond cubic materials would be composed of arrays of two lattice dislocations. To date, only these two structural units have been seen in these boundaries, but rarely in his proposed combinations.[20]

Figure 2 shows the boundary structure, formed at a relatively low tilt angle of $16^{\circ}(\Sigma=25$ $\{170\}<001>$ ) consisting of isolated defect cores separated by perfect crystal units. These defect cores are seen to be asymmetric combinations of four identical units. In this $<001\rangle$ projection, the individual units appear as a pentagon with an attached triangle. The closure failure around the entire defect is $a[100]$. This is the net Burgers vector and defect spacing expected for a $16^{\circ}$ tilt boundary. However, the defect core appears to be more complex than is necessary. There are twice the number of dislocations needed for this tilt angle and the defects are clustered rather than uniformly spaced.

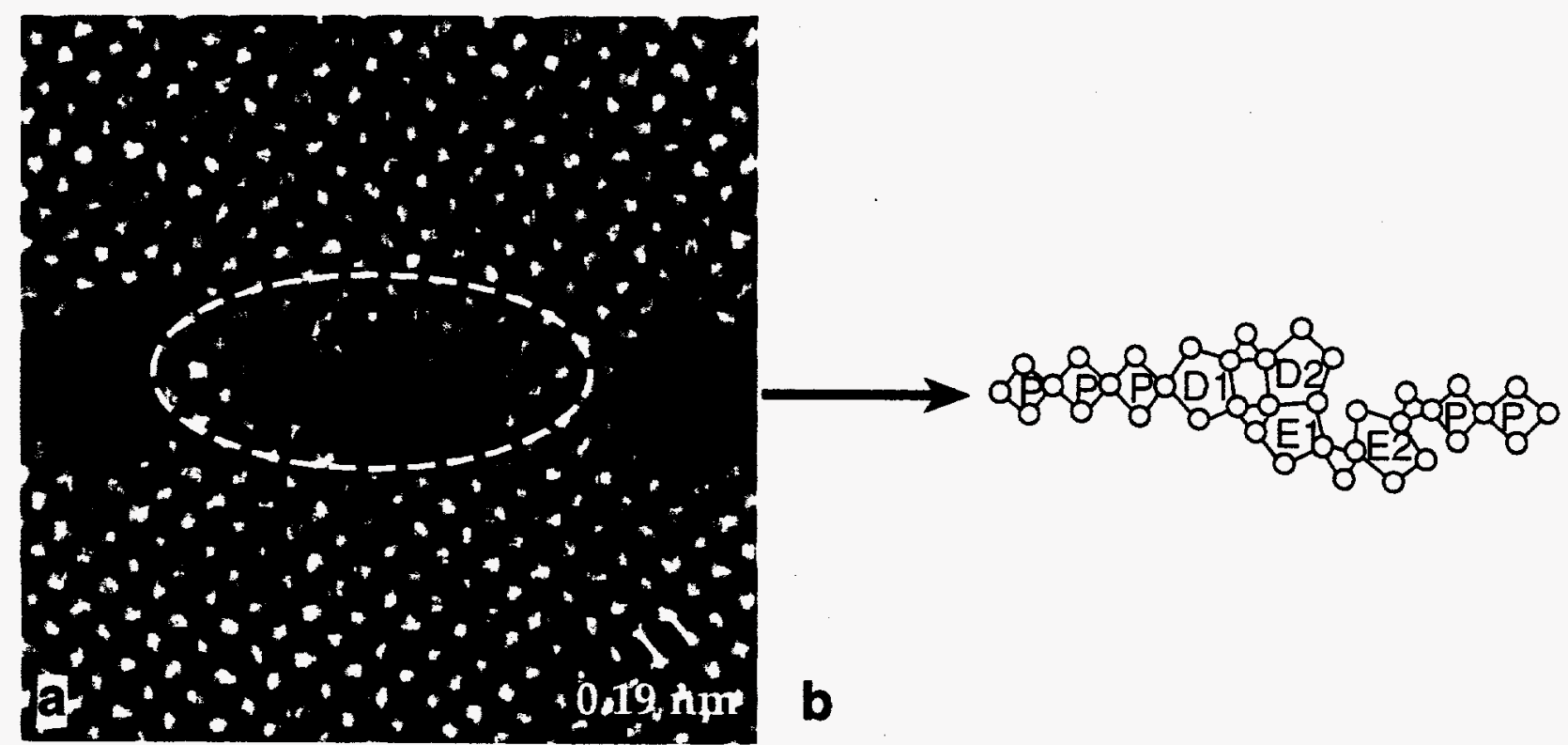

Figure 2. (a) Z-contrast image of a symmetric $16^{\circ} \Sigma=25\{170\}<001>$ silicon tilt grain boundary as viewed along the bicrystal's common $<001>$ direction. The boundary is seen to consist of a periodic array of defect cores separated by relatively undisturbed crystal units. As seen in the schematic (b) obtained directly from the image, the defect core consists of four identical units that in this projection appear as a connected pentagon-triangle pair. The units marked D1 and D2 are a connected dislocation dipole. The units marked E1 and E2 are the dislocations that accommodate the misorientation of the two grains. 
The boundary actually contains two of these asymmetric defect cores. The second is related to the first by a mirror across the $\{170\}$ boundary plane and a glide. It is also found that these two asymmetric defect cores have created somewhat of a dilemma for the boundary. The resulting structure is a compromise between uniform spacing of the defect cores and maintaining a planar boundary.

When using the Z-contrast technique, bright features can be directly interpreted as positions of atomic columns. However, because the image is a two dimensional projection, there is no information on the relative vertical positions of atoms in different columns. Molecular dynamics simulations, using the two dimensional coordinates extracted from the image, were used to determine the three dimensional atomic arrangement present in the boundary. All the features of the image were reproduced in the simulation. From this three dimensional structure, the four units are found to be a single dislocation type - an edge dislocation with Burgers vector $\mathbf{b}=a / 2$ [110] with a [001] line direction. Again, we find the $<001>$ boundary cores can be described as arrays of closely spaced dislocations. Interestingly, only two of these dislocations are required to accommodate the misorientation of the two grains. The other two unexpected units are arranged as a connected dislocation dipole, with equal but opposite Burgers vectors and, therefore, are geometrically unnecessary. It appears that the energy of this boundary can be decreased with the addition of these extra or redundant dislocations.

Increasing the tilt angle to $23^{\circ}(\Sigma=13\{150\}<001>)$ produces another unforeseen atomic arrangement in which the entire boundary is composed of defect units (Fig. 3). If the bulk silicon positions are stripped from the image, the grain boundary core consisting of a continuous string of pentagonal and triangular features remains. This $<001>$ projection has been used to calculate the three dimensional structure of the defect. From the calculated grain boundary core, two distinct structural units can be identified. These two units have the same atomic configuration as two known diamond cubic lattice dislocations. It is noteworthy that these dislocation cores remain distinct; they have the same atomic configuration as they would isolated in the bulk crystal. The boundary dislocations do not mix or collapse even when spaced every $0.23 \mathrm{~nm}$ along the boundary.
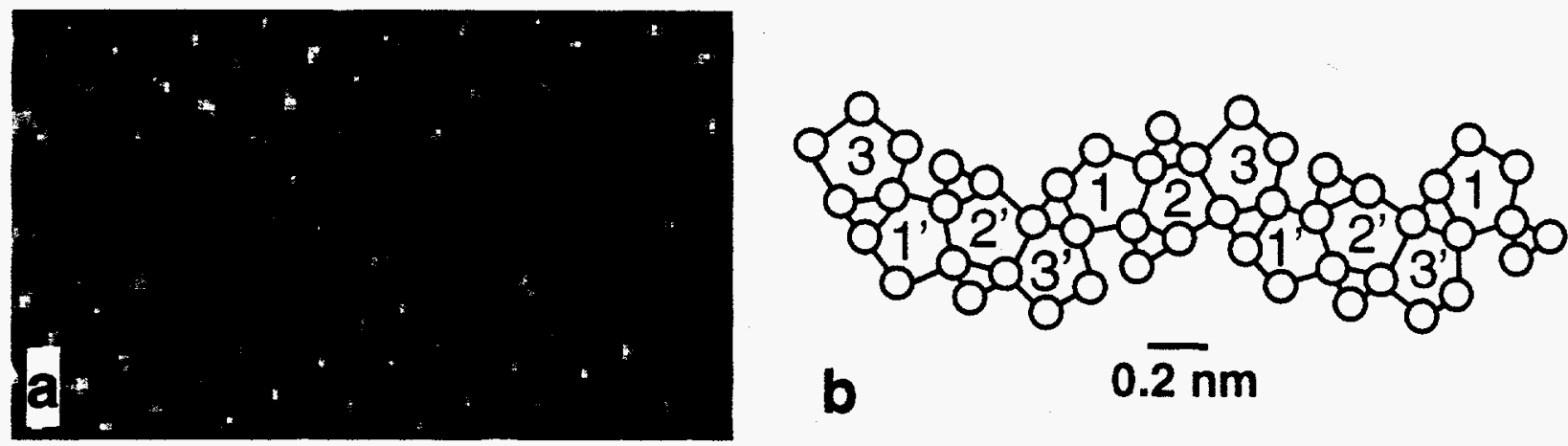

Figure 3. Z-contrast image of a symmetric $23^{\circ} \Sigma=13\{150\}<001>$ silicon tilt grain boundary and the projected atomic column positions obtained directly from the image. The boundary is seen to be periodic with a $1.38 \mathrm{~nm}$ repeat, containing two distinct units (edge dislocations, 1 and 1', and mixed dislocations, 2,2',3, and $3^{\prime}$ ) arranged in a contiguous sequence of six dislocation cores. The bright features in the grains are separated by $0.19 \mathrm{~nm}$.

The line direction of both dislocations is parallel to the boundary tilt axis. The units marked 1 and $1^{\prime}$ in the schematic, (Fig. $3 b$ ) are the edge dislocations seen in the $16^{\circ}$ boundary. The units marked 2, 2', 3, and $3^{\prime}$ are mixed dislocations with equal edge and screw components. Their Burgers vector is inclined $45^{\circ}$ to the $(001)$ plane. It is the presence of these mixed dislocations, arranged as dipoles, that is the unforeseen feature of this boundary. As in the $16^{\circ}$ boundary, the dipoles could be replaced by units of perfect crystal. The reason for these extra boundary dislocations is not obvious from classical potential calculations that indicate, while the strain energy in the 
boundary is more uniform when the dipoles are present, there is little influence on the total boundary energy. We await first principles calculations to further explore this mystery.

The investigation of $<001\rangle \mathrm{Si}$ tilt boundaries using Z-contrast imaging has significantly reduced the perceived complexity of these defects. We have found no evidence of multiple grain boundary structures coexisting in any of the boundaries we have examined. However, the mystery of the redundant dislocations remains to be solved, for the goal remains to be able to predict the boundary structures without having to actually examine them.

\section{Intrinsic electronic structure of grain boundaries}

It is well known that the electrical properties of polycrystalline silicon are dominated by localized electronic states in the band gap and that these localized states are associated with the presence of grain boundaries.[21,22] However, most previous calculations of the electronic structure of clean semiconductor ( $\mathrm{Si}$ or $\mathrm{Ge}$ ) boundaries have found no gap states associated with the boundary core and, thus, concluded that the specific intrinsic boundaries were electrically inactive.[23-27] These calculations were done on pure tilt boundaries in which all atoms retain fourfold coordination. We, too, have found using tight-binding calculations on our experimentally observed atomic configurations that there are no states in the band gap for the three tilt boundaries discussed above (see Fig. 4). Experimental measurements on high-angle grain boundaries in zone-melt-recrystallized films confirm that clean silicon tilt boundaries are electrically inactive even after long high-temperature anneals.[28]

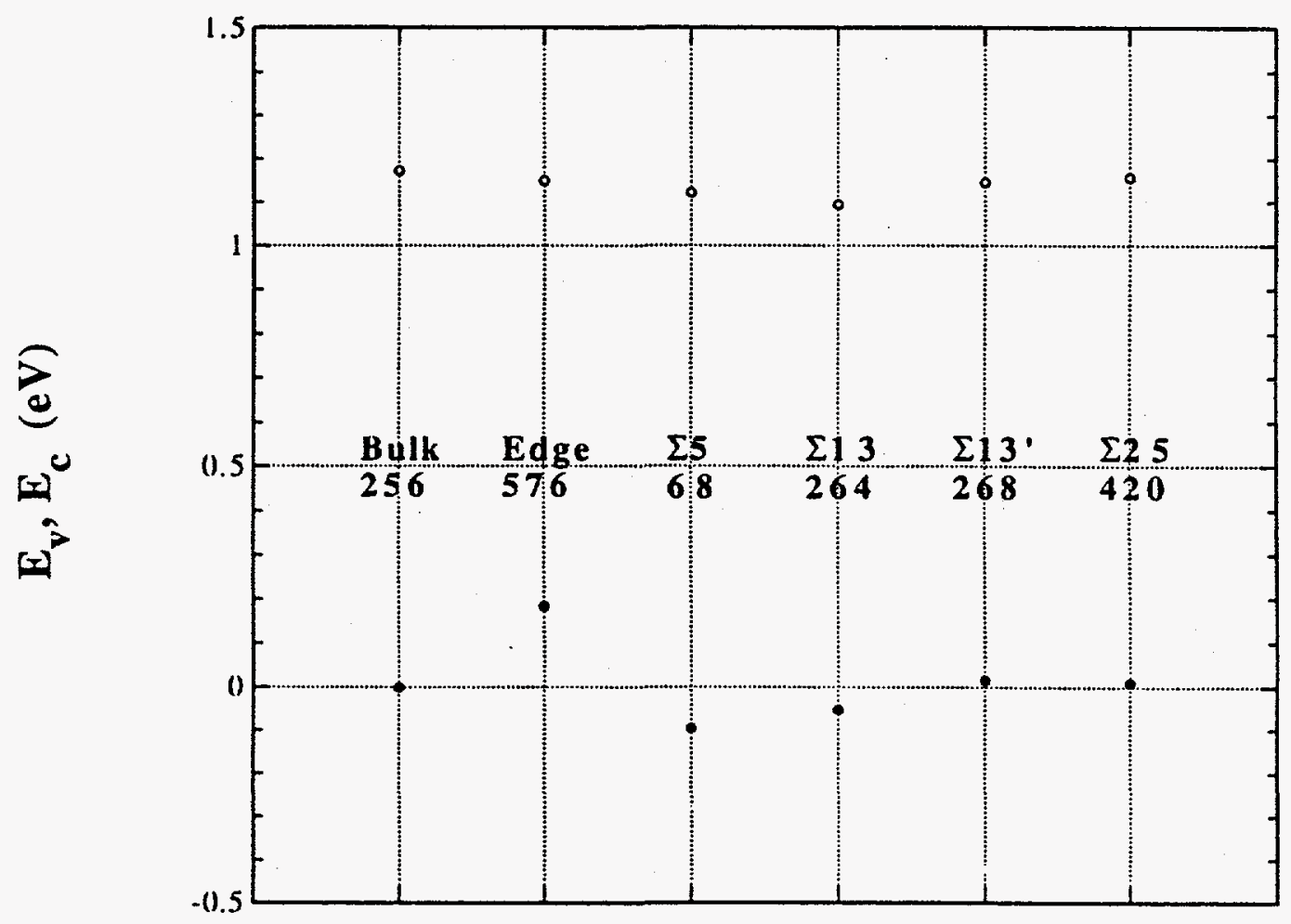

Figure 4. Valence band maximum, $\mathrm{E}_{\mathrm{v}}$, and conduction band minimum, $\mathrm{E}_{\mathrm{c}}$, for various defects in silicon: an edge dislocation dipole in a 576-atom supercell, a 68-atom supercell containing two $37^{\circ}$ $<001>$ tilt boundaries, a 264-atom supercell containing two $23^{\circ}<001>$ tilt boundaries, a 268 -atom supercell containing an alternative $23^{\circ}<001>$ tilt boundary structure, and a 420 -atom $16^{\circ}<001>$ tilt boundary supercell. Only the isolated edge dislocations produce states in the band gap of bulk silicon. 
More recently, tight-binding simulations of higher energy twist boundaries in silicon have been examined.[29] These simulations showed that, although it was possible to produce structures of four different twist boundaries that did not contain coordination defects, the boundaries contain larger bond distortions and much larger interfacial energies than found for tilt boundaries. The associated electronic structure was found to contain band gap tail states up to $0.17 \mathrm{eV}$ above the valence band maximum of bulk silicon and states below the conduction band edge minimum by about $0.01 \mathrm{eV}$. This study demonstrated that structural disorder (coordination defects are not necessary) can generate electronic states penetrating into the band gap as seen experimentally. One problem with this connection between theory and experiment is that the measured density of defect states associated with grain boundaries $\left(10^{10} \mathrm{~cm}^{-2}\right.$ to $\left.10^{12} \mathrm{~cm}^{-2}\right)$ would suggest a separation between point defects in an electrically active boundary of 10 to $100 \mathrm{~nm}$. This is much larger than the distance between the highly distorted bonds in the structures calculated for silicon twist boundaries. Thus, it is still necessary to determine, firstly, the actual structures of silicon twist boundaries and, secondly, if there is another source of electrical activity in intrinsic boundaries. The first problem is difficult to address because direct experimental atomic structure determinations have only been done using transmission electron microscopy on tilt boundaries. The actual structure of twist boundaries in silicon remain to be determined by some technique other than transmission electron microscopy. We have begun to address the second problem by examining the electrical structure of isolated $\langle 110\rangle$ edge dislocations, a "building block" used in $<110>$ tilt boundaries. [30]

\section{Intrinsic electronic structure of dislocations}

Using a combination of LDA and tight-binding calculations, the electronic structure of two types of edge dislocations found in silicon, the $(a / 2)<110>$ perfect edge dislocation and the $(a / 6)$ $<112>90^{\circ}$ Shockley partial dislocation have been examined[30] (see Fig. 5). The calculations were performed using periodic supercells containing a dislocation dipole, i.e., two separated dislocations with equal but opposite closure failures. The supercell size (and hence the dislocation separation) ranged from 32 to 576 atoms. The main effect of the dislocations was to push the top of the valence band into the gap, and this effect is enhanced as the dislocation separation increases. At the $\Gamma$ point, the shift in the top of the valence bands for the perfect edge dislocation dipoles changes from $0.01 \mathrm{eV}$ for a $0.7 \mathrm{~nm}$ separation to $0.18 \mathrm{eV}$ for separations greater than $2.2 \mathrm{~nm}$. It is found that the charge density for the top most valence band is concentrated at the apexes of the fivefold rings in the dislocation cores where the bonds are most compressed and the bond angles are the smallest. We found similar but weaker effects for the $90^{\circ}$ partial, as expected, since the strain varies to first order with the magnitude of the Burgers vector. For the partial dislocation, the upward shift of the top of the valence band was found to increase to $0.1 \mathrm{eV}$ for dislocation separations greater than $2.0 \mathrm{~nm}$.

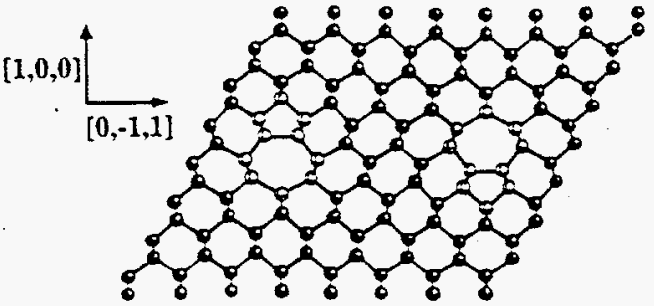

a

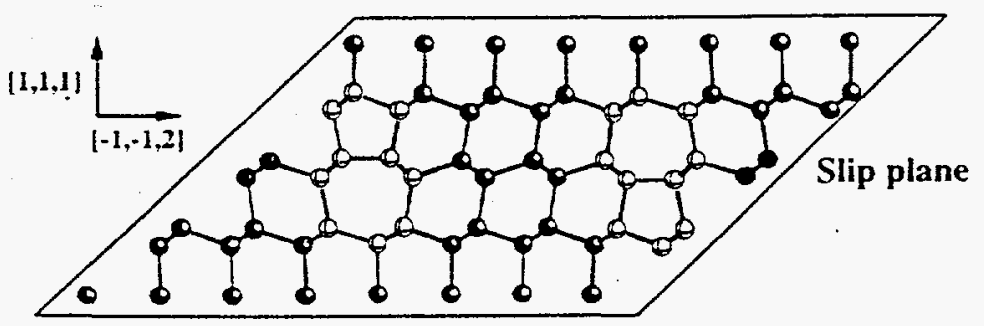

b

Figure 5. $<110>$ projections of the atomic configurations of two edge dislocations in silicon. All atoms retain fourfold coordination even in the core of the (a) $(a / 2)<110>$ perfect edge dislocation and (b) the $(a / 6)<112>90^{\circ}$ Shockley partial dislocation.

This result is theoretical evidence that dislocation cores with no dangling bonds and no impurities can, in their intrinsic state, be electrically active. The enhancement of the band-edge shift 
correlates well with the increased localization of the structural distortions in the dislocation cores that occurs with increased separation of the dislocations. If the dislocation cores are too close, there is substantial overlap of the strain fields, the lattice distortions become more uniformly distributed, and no gap states are produced.[31]

Thus, the uniformly and closely spaced arrays of dislocations seen in symmetrical high angle tilt boundaries, would not be expected to produce gap states. However, any boundary that deviates from these special orientations will contain additional dislocations (so-called secondary dislocations) that will have larger separations. These additions to the primary dislocation structure of the boundary would be expected to produce gap states at the valence band edge.

Neither dislocation produced much of an effect at the conduction band edge. This finding is consistent with theoretical studies on model $\mathrm{SiO}_{2} / \mathrm{Si}$ interfaces in which it was found that distortions of $\mathrm{Si}-\mathrm{Si}$ bonds result in valence band tails. Distortions of the $\mathrm{Si}-\mathrm{O}-\mathrm{Si}$ angle were responsible for the tail states at the conduction band and broken bonds resulted in midgap states.[32] It is also consistent with calculations of model silicon twist boundaries that found an asymmetric introduction of states into the band gap with up to $0.17 \mathrm{eV}$ valence band tails and smaller $(\sim 0.01 \mathrm{ev})$ conduction band tails.[29]

\section{Impurities in grain boundaries}

The discussion above has concentrated on the intrinsic configurations and electronic structure of silicon grain boundaries. We now ask what impurities do when segregated to grain boundaries by looking at one dopant (arsenic) and a common impurity (oxygen).

Arsenic. [33] Impurities can interact with dislocations and grain boundaries both elastically and electrically. One impurity of interest in Si is arsenic, a n-type dopant, which when segregated to the grain boundaries, reduces the free carrier concentration of the semiconductor. Figure 6 is a $\mathrm{Z}$ contrast image of a $23^{\circ} \Sigma=13\{150\}<001>$ Si tilt boundary doped with arsenic. It can be seen the boundary contains a periodically repeating cluster of three relatively bright atomic columns. These columns are on average $\sim 20 \%$ brighter than their neighbors, which corresponds to $\sim 5$ at. $\%$ arsenic in these columns if it is assumed that the scattered intensity is proportional to the atomic number squared. Thus, the compositional sensitivity of the Z-contrast technique allows the detection of one or two arsenic atoms in a column of approximately 40 silicon atoms. Our first-principles calculations provided both support and further explanation of the images.[34] We found that single arsenic atoms will segregate at the $<001>$ tilt boundaries, $[33,34]$ however, they showed little preference of one site over another. The bright features seen at the boundary are, therefore, most likely not due to isolated impurities. Interestingly, the calculations indicate paired arsenic impurities have a larger segregation energy than isolated dopants.[33] The reason for this is that the arsenic dimers in the grain boundary can push or be pulled apart to attain their preferred threefold coordination. The calculations also indicate that the observed segregation site is preferred by isolated pairs of dopants. Filled arsenic dimer columns favor another site.
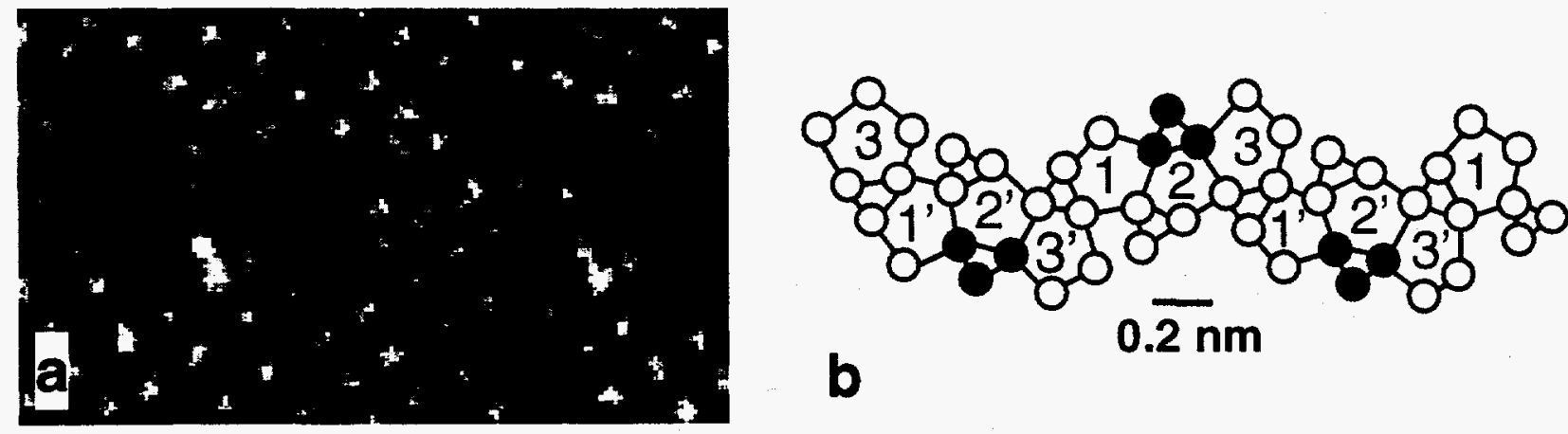

Figure 6. Z-contrast image of a symmetric $23^{\circ} \Sigma=13\{150\}<001>$ silicon tilt grain boundary doped with arsenic. The columns containing segregated arsenic (indicated with gray in the schematic) are found to be significantly brighter than the other triangular features in the boundary. These bright features, which are repeated every $0.69 \mathrm{~nm}$ are not observed in the undoped bicrystal (Fig. 3). 
We have examined the electronic energy levels that result from As segregated to a dislocation core.[35] As expected, isolated arsenic atoms have a shallow donor level below the conduction band edge. However, when segregated in its preferred configuration of chains of As dimers, midgap states are formed. These are lone-pair states that As atoms have when they are threefold coordinated. Because of the close proximity of threefold coordinated As atoms, these lone-pair states split, with half in the band gap and half in the valence band.

Interestingly, we have also found that impurities can induce a structural transformation of both dislocations [36] and grain boundaries [34] in silicon. This highlights the importance of being able to not only directly image the structure of the defects but also to be able to detect the presence of impurities. Extended defects have a propensity to attract contaminates and the impurities may be responsible for the observed atomic arrangements.

Oxygen. Silicon wafers grown by the Czochralski method typically contain $\sim 10^{18} / \mathrm{cm}^{3}$ oxygen atoms. Before thermal processing, these impurities are generally in electrically inactive twofold coordinated interstitial sites.[37] Carefully devised thermal treatments and tightly specified oxygen contents are now used to generate $\mathrm{SiO}_{2}$ precipitates that serve to bind metallic impurities away from the near-surface regions of the wafers.[38] However, under certain annealing conditions oxygencontaining defects can form that are double shallow donors, known as thermal donors. Since their discovery [39] in 1954, annealing procedures have been developed to avoid the presence of these electrically active defects in single crystal wafers. While electrical activity of boundaries in cast silicon has been linked to the presence of oxygen,[40-42] a microscopic mechanism for the activity had not been proposed. We have begun an investigation of oxygen in a silicon grain boundary using LDA calculations [42] and found that individual oxygen atoms will bind at a $\left.37^{\circ}<001\right\rangle$ silicon tilt boundary with a segregation energy of $0.35 \mathrm{eV}$. The oxygen atom is incorporated in a twofold interstitial position and is electrically inactive. Additional $\mathrm{O}$ atoms can lower their energy by forming staggered Si-O-Si chains similar to those predicted in bulk silicon.[43,44] In bulk silicon, three O atoms in staggered chains is the lowest energy structure, whereas an alternative structure is metastable and electrically active. $[43,44]$ This electrically active defect has two electrons that occupy very diffuse bound states at the conduction band edge $\left(E_{c}-\sim 100 \mathrm{eV}\right)$. These calculations confirmed earlier suggestions [45] that this structure represents the core of thermal donors. We find that, in the grain boundary, the thermal donor configuration has essentially the same energy as the staggered chains (see Fig. 7). The predicted segregation of thermal donors in the grain boundaries offers a possible explanation for the oxygen-related electrical activity of boundaries in cast silicon.

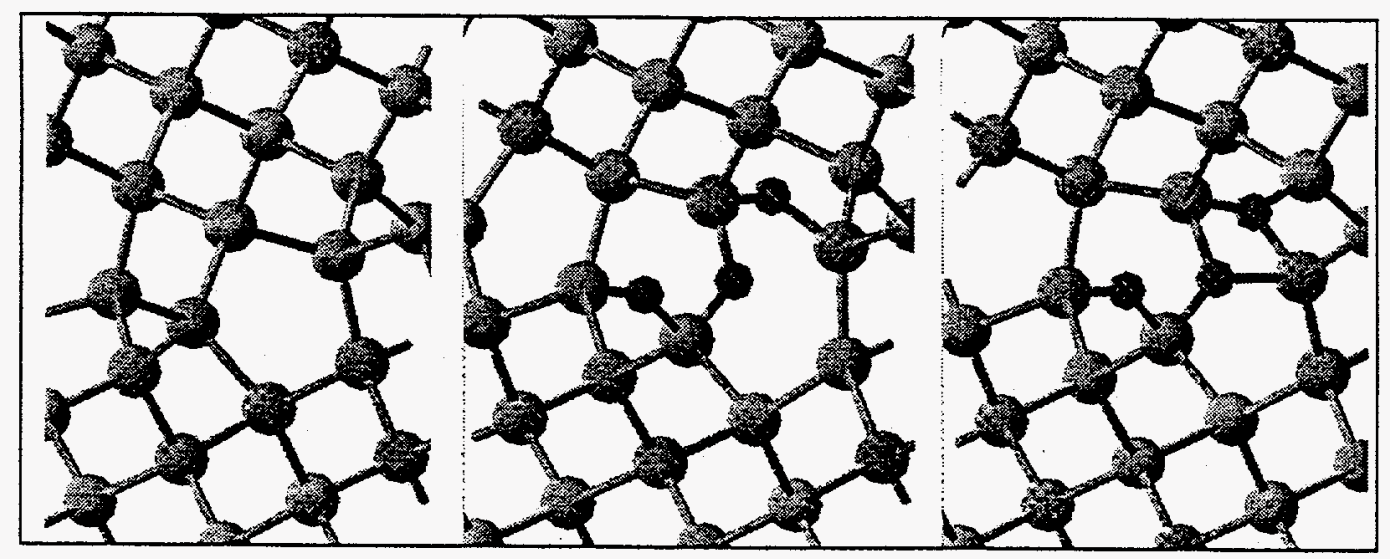

(a)

(b)

(c)

Figure 7. $<001>$ projections of a symmetric $37^{\circ} \Sigma=5\{130\}<001>$ silicon tilt grain boundary. (a) shows the intrinsic ground state configuration. (b) shows the electrically inactive $\mathrm{O}_{3}$ cluster in which all $\mathrm{O}$ atoms are twofold coordinated and all $\mathrm{Si}$ atoms are fourfold coordinated. (c) shows a cluster in which two of the three $O$ atoms are threefold coordinated resulting in diffuse states at the conduction band edge. 
Although larger complexes have not yet been examined, we believe that the behavior of oxygen in the grain boundary is similar to that in bulk silicon. In calculations of four $\mathrm{O}$ atoms in bulk silicon, [46] it was found that a metastable structure is formed in which a silicon atom is surrounded by four $\mathrm{O}$ atoms. If this central silicon atom is removed, the remaining structure has fourfold coordinated silicon atoms, twofold coordinated oxygen atoms, and is electrically inactive. This $\mathrm{SiO}_{2}-$ like structure is the lowest energy state for four $\mathrm{O}$ atoms in $\mathrm{Si}$, indicating that even though any number of $\mathrm{O}$ atoms can lower their energy by forming staggered $\mathrm{Si}-\mathrm{O}-\mathrm{Si}$ chains, the four $\mathrm{O}$ atom chain will prefer to transform into a $\mathrm{SiO}_{2}$-like cluster by ejecting a $\mathrm{Si}$ atom. This explains why large staggered chains have not been observed; they are metastable and transform into $\mathrm{SiO}_{2}$-like precipitates. The predicted reaction is also consistent with the observation that the disappearance of the electrical activity (thermal donors) is accompanied by the emission of Si interstitials.[47]

Chadi also investigated interactions of a few impurities with these $\mathrm{O}$ complexes and found $\mathrm{H}$, $\mathrm{B}, \mathrm{N}$, and $\mathrm{Al}$ would segregate to these defects. [45] It is possible that these thermal donors are not only responsible for shallow donor states, but they may also be involved in the formation of midgap states by gettering metallic impurities.

\section{Summary}

Directly interpretable Z-contrast images have been combined with first principles calculations to provide atomic scale information on the geometric and electronic structures of extended defects in silicon. It was found that the $\langle 110\rangle$ and $<001\rangle$ tilt boundaries examined are composed of structural units that are identical to isolated dislocations. These units are not mixing or collapsing to form new structures even when spaced every $0.23 \mathrm{~nm}$ along the boundary. Redundant dislocations, arranged as a connected dislocation dipole, with equal but opposite Burgers vectors are found in $<001>$ tilt boundaries in silicon. These structures are geometrically unnecessary to accommodate the misorientation, they have no detectable effect on the calculated (using classical potentials) excess energy of the boundary, and they have no effect on the density of states in the band gap region of silicon.

From calculations of the electronic structure of isolated dislocations, we propose that secondary dislocations, even though they have no dangling bonds, can produce gap states extending up to $0.2 \mathrm{eV}$ beyond the top of the valence band edge of bulk silicon. We found oxygen atoms will segregate to grain boundaries and form clusters that produce shallow donor states $\sim 100 \mathrm{meV}$ below the bottom of the conduction band edge.

Arsenic will segregate to grain boundaries and it prefers to form arsenic dimers in the boundary. These paired atoms can more easily more away from each other in the boundary to attain their preferred threefold coordination (leaving its Si neighbors fourfold coordinated). The threefold coordinated arsenic produces midgap states in silicon.

\section{Acknowledgments}

This work was performed at Oak Ridge National Laboratory, which is managed by Lockheed Martin Energy Research Corp., under DOE Contract No. DE-AC05-960R22464, ORN Grant No. N00014-95-1-0906 and by the ORNL Postdoctoral Research Associates Program administered by ORNL and ORISE.

\section{References}

* on leave from the Institute of Physics, Polish Academy of Sciences, 02-668 Warsaw, Poland.

[1] H. R. Huff, D. W. McCormack, Jr., C. Au, T. Messina, K. Chan, and R. K. Goodall, Jpn. J. Appl. Phys. 37, 1210 (1998).

[2] H. J. Queisser and E. E. Haller, Science 281, 945 (1998).

[3] P D Nellist and S J Pennycook, J. Microsc. 190, 159 (1998), P D Nellist and S J Pennycook, Ultramicroscopy (submitted).

[4] Lord Rayleigh, Phil. Mag. 42, 167, (1896).

[5] S. J. Pennycook and D. E. Jesson, Acta metall. mater. 40, S149 (1992).

[6] D. E. Jesson and S. J. Pennycook, Proc. Roy. Soc Lond. A 449, 272 (1995).

[7] P. Hohenberg and W. Kohn, Phys. Rev. 136, B864 (1964).

[8] G. P. Kerker, J. Phys. C 13, L189 (1980).

[9] H. J. Monkhorst and J. D. Pack, Phys. Rev. B 13, 5188 (1976). 
[10] M. C. Payne, M. P. Teter, D. C. Allan, T. A. Arias, and J. D. Joannopoulos, Rev. Mod. Phys. 64, 1045 (1992).

[11] P. Vogl, H. P. Hjalmarson and J. D. Dow, J. Phys. Chem. Solids 44, 365 (1983).

[12] G. Quincke, Proc. Roy. Soc. A76, 431 (1905).

[13] G. I. Taylor, Proc. Roy Soc. 145A, 388 (1934).

[14] J. M. Burgers, Proc. Kon. Ned. Akad. V. Wet. Amsterdam 42, 293 (1939).

[15] A. P. Sutton and V. Vitek, Phil. Trans. Roy. Soc. Lond. A 309, 1 (1983); 309, 37; 309, 55 (1983).

[16] G. H. Bishop and B. Chalmers, Scripta metall. 2, 133 (1968).

[17] O. L. Krivanek, S. Isoda, and K. Kobayashi, Phil. Mag. 36, 931 (1977).

[18] J. Hornstra, J. Phys. Chem. Solids 5, 129 (1958).

[19] J. Hornstra, Physica 25, 409 (1959).

[20] A. Bourret and J. L. Rouviere, in Polycrystalline Semiconductors, edited by H. J. Moller, H. P. Strunk, and J. H. Werner, Springer Proc. Phys. 35, 8 (1989).

[21] C. R. M. Grovenor, J. Phys. C 18, 4079 (1985).

[22] J. H. Werner, in Structure and Properties of Dislocations in Semiconductors 1989, edited by S. G. Roberts et al., Inst. Phys. Conf. Series 104 (Institute of Physics, Bristol, 1989) p 63.

[23] R. E. Thomson and D. J. Chadi, Phys. Rev. B 29, 889 (1984).

[24] D. P. DiVincenzo,O. L. Alerhand, M. Schluter, and J. W. Wilkins, Phys. Rev. Lett. 56, 1925 (1986).

[25] M. Kohyama, R. Yamamoto, Y. Ebata, and M. Kinoshita, J. Phys. C 21, 3205 (19.88).

[26] A. T. Paxton and A. P. Sutton, J. Phys. C 21, L481 (1988).

[27] T. A. Arias and J. D. Joannopoulos, Phys. Rev. B 49, 4525 (1994).

[28] P. V. Evans, D. A. Smith, and C. V. Thompson, Appl. Phys. Lett. 60, 439 (1992).

[29] M. Kohyama and R. Yamamoto, Phys. Rev. B 49, 17102 (1994); M. Kohyama and R. Yamamoto, Phys. Rev. B 50, 8502 (1994).

[30] F. Liu, M. Mostoller, V. Milman, M. F. Chisholm, and T. Kaplan, Phys. Rev. B 51, 17192 (1995).

[31] J. R. K. Bigger, D. A. McInnes, A. P. Sutton, M. C. Payne, I. Stich, R. D. King-Smith, D. M. Bird, and L. J. Clarke, Phys. Rev. Lett. 69, 2224 (1992).

[32] R. B. Laughlin, J. D. Joannopoulos, and J. D. Chadi, in The Physics of $\mathrm{SiO}_{2}$ and its Interfaces, edited by S. T. Pantelides (Pergamon Press, New York, 1978) p 321.

[33] M. F. Chisholm, A. Maiti, S. J. Pennycook, and S. T. Pantelides, Phys. Rev. Lett. 81, 132 (1998).

[34] A. Maiti, M. F. Chisholm, S. J. Pennycook, and S. T. Pantelides, Phys. Rev. Lett. 77, 1306 (1996).

[35] A. Maiti, T. Kaplan, M. Mostoller, M. F. Chisholm, S. J. Pennycook, and S. T. Pantelides, Appl. Phys. Lett. 70, 336 (1997).

[36] T. Kaplan, M. Mostoller, and M. F. Chisholm, Phys. Rev. B in press (1998).

[37] G. Obermeier, J. Hage, and D. Huber, J. Appl. Phys. 82, 595 (1997).

[38] G. D. Watkins and J. W. Corbett, Phys. Rev. 121, 1001 (1961).

[39] C. S. Fuller, J. W. Dietzenberger, N. B. Hannay and E. Buehler, Phys. Rev. 96, 833 (1954).

[40] D. Redfield, Appl. Phys. Lett. 38, 174 (1981).

[41] L. L. Kasmerski, J. Vac. Sci. Technol. 20, 423 (1982).

[42] P. E. Russell, C. R. Herrington, D. E. Burke, and P. H. Holloway, in Grain Boundaries in Semiconductors ed. H. J. Leamy, G. E. Pike, and C. H. Seager (North-Holland, New York, 1982) p 185.

[43] R. Buczko, M. F. Chisholm, and S. T. Pantelides, unpublished research.

[44] M. Needels, J. D. Joannopoulos, Y. Bar-Yam, and S. T. Pantelides, Phys. Rev. B 43, 4208 (1991).

[45] D. J. Chadi, Phys. Rev. Lett. 77, 861 (1996).

[46] S. T. Pantelides and M. Ramamoorthy, in Silicon-on-Insulator Technology and Devices III, ed. by S. Cristoloveanu et al. (Electrochemical Society, Pennington, N.J., 1996).

[47] R. C. Newman, A. S. Oates, and F. M. Livingston, J. Phys. C 16, 1667 (1983); M. Claybourn and R. C. Newman, Appl. Phys. Lett. 51, 2197 (1987). 